Check for updates

Cite this: RSC Adv., 2018, 8, 36662

Received 30th September 2018 Accepted 23rd October 2018

DOI: $10.1039 / c 8 \mathrm{ra0} 8135 b$

rsc.li/rsc-advances

\section{Computational investigation of the control of the thermodynamics and microkinetics of the reductive amination reaction by solvent coordination and a co-catalyst $\uparrow$}

\begin{abstract}
Esra Boz, (D) ab Nurcan Ş. Tüzün ${ }^{(\mathbb{D})}{ }^{b}$ and Matthias Stein (D) *a
Amines are among the most important and frequently used chemical compounds due to their biological activity and a wide range of applications in industry. Reductive amination reactions are an efficient and facile route to synthesize long chain amines from sustainable sources by using a different available aldehydes and ketones, and a large variety of amines including primary, secondary and tertiary forms. The pathway of the reaction process is critically dependent on reaction parameters such as the $\mathrm{pH}$ of the reaction medium, choice of solvent (explicitly coordinating solvent) and process conditions. These parameters are affecting the reaction performance and the selectivity but are still not fully rationalized. Here, we investigate the microkinetics and thermodynamics of the individual steps of the reductive amination reaction by exploring the systems' parameters. Explicit water coordination to the aldehyde leads to a stepwise rather than concerted nucleophilic addition with a lower activation barrier by 6 $10 \mathrm{kcal} \mathrm{mol}{ }^{-1}$. At low $\mathrm{pH}$, the pathway is changed by a direct protonation of the amine substrate. This protonation does not strongly affect the kinetics of the reaction, but the thermodynamic equilibria. The presence of an acid as a co-catalyst leads to the formation of an iminium intermediate and this drives the reaction forward. Thus, the presence of an acid as a co-catalyst clearly renders this pathway the thermodynamically preferred one. Consequently, altering the reaction parameters does not only influence the reaction kinetics, but also the thermodynamic profile of the pathways in all cases. Further understanding of the reaction dynamics is essential to develop a microkinetic model of the reaction to then control and engineer the process in order to rationally design routes to tailor-made products.
\end{abstract}

\section{Introduction}

A green chemistry approach to the production of fine chemicals and the utilization of natural products from sustainable sources is becoming more significant in industrial applications. Amines represent an important class of high-quality chemicals which are used as intermediates in a range of applications including pharmaceuticals, agricultural chemicals, rubber chemicals, water treatment chemicals, and also as solvents. ${ }^{1}$ The transformation of industrially available fatty acids from sustainable sources into long chain amines via an environmentally friendly and sustainable reaction has recently gained significant importance (Scheme 1). ${ }^{2}$ Indeed, the reaction of reductive amination has been recognized to be one of the most relevant challenges for process design for the pharmaceutical industry. ${ }^{3}$

${ }^{a}$ Max Planck Institute for Dynamics of Complex Technical Systems, Molecular Simulations and Design Group, Magdeburg, Germany. E-mail: matthias.stein@ mpi-magdeburg.mpg.de

${ }^{b}$ Istanbul Technical University, Department of Chemistry, Istanbul, Turkey

$\dagger$ Electronic supplementary information (ESI) available. See DOI: $10.1039 / \mathrm{c} 8 \mathrm{ra0} 0135 \mathrm{~b}$
The sequential or tandem coupling of industrial chemical processes $^{4}$ gives an elegant access to these classes of compounds, for example by coupling a hydroformylation reaction which is followed by an amination of the obtained aldehyde and finally a hydrogenation of the enamine to produce saturated, long chain primary, secondary or tertiary amines. ${ }^{5}$ Starting from $n$-undecene, first $n$-dodecanal (lauryl aldehyde, a fragrance) is being produced which is then converted into $N, N$ diethyltridecane-1-amine $\left(\mathrm{C}_{15} \mathrm{H}_{33} \mathrm{~N}, \quad\right.$ an anti-infective; Scheme 1).

Such a facile and efficient route for a hydroaminomethylation (HAM) process is of great interest to the chemical and pharmaceutical industry. ${ }^{6,7}$

The reductive amination initiates with the addition of an amine to the carbonyl group of the aldehyde/ketone and a hemiaminal as intermediate is formed (Scheme 2). The subsequent condensation reaction results in an imine or an iminium ion depending on the $\mathrm{pH}$ of the reaction medium. The equilibrium between aldehyde/ketone and imine can be controlled by continuous removal of the released water. At the final step, the desired amine is obtained by a reduction of the 


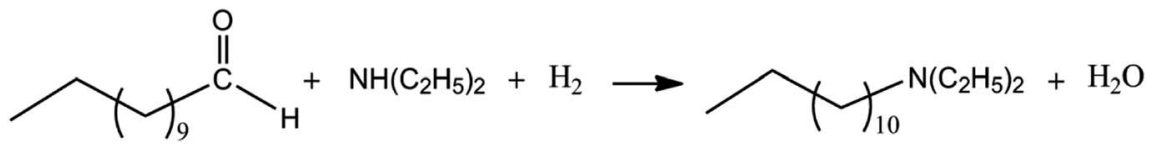

Scheme 1 Production of long chain diethylamines from aldehydes via reductive amination.

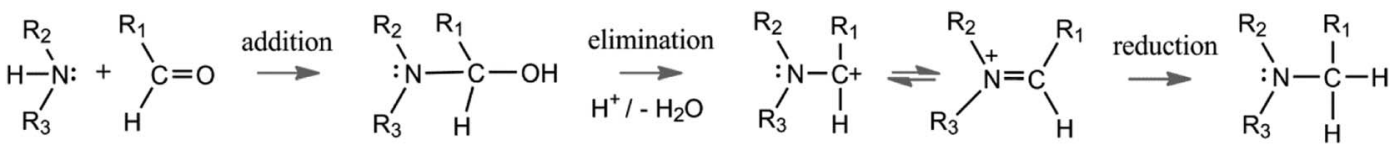

Scheme 2 Proposed mechanism for the reductive amination of aldehydes.

$\mathrm{C}=\mathrm{N}$ bond by using either an organic hydride donor or a transition metal catalyst. However, reduction of the $\mathrm{C}=\mathrm{N}$ bond in an efficient, industrially applicable and non-toxic way is still an area of intense research.

Due to their great importance, there are numerous synthetic approaches to form amines. These methods are generally classified as 'direct' and 'indirect' according to the instance of addition of the reducing agent. ${ }^{8}$ The reaction is described as 'direct', when aldehyde, amine and reducing agent are reacting in a one pot process. ${ }^{5}$ The drawback of the direct reduction process is the competition between reduction of the $\mathrm{C}=\mathrm{O}$ carbonyl group of the aldehyde and the $\mathrm{C}=\mathrm{N}$ unsaturated bonds of imine which leads to a number of side products. Thus, the appropriate choice of the reducing agent becomes more critical. In order to obtain an amine as a final product, the reducing agent must react selectively with the imine (or enamine, when secondary amines are used) or iminium ion rather than the carbonyl group of the aldehyde. Usually, direct reductive amination is preferred over an 'indirect' process, because it is more conveniently handled and a more efficient process, especially in large scale applications. ${ }^{4}$ The use of borohydrides, for example sodium triacetoxyborohydride, ${ }^{8-10}$ is one example of a simple and cheap choice of reducing agents because of its different selectivity at different $\mathrm{pH}$, its stability in acidic conditions $(\mathrm{pH}=3)$ and its solubility in polar solvents. However, the toxicity of the reducing agent and its low conversion rate in case of unreactive ketones hamper the large scale application. ${ }^{10}$ Recently, a silane-based reductant was reported for the direct reductive amination to give only one representative of the organosilane family. ${ }^{11}$ This reducing reagent is not only cheap and metal free to classify it as a green process; but it also shows a high selectivity to the $\mathrm{C}=\mathrm{N}$ bond which prevents the formation of undesired side products.

Heterogeneous catalytic reductive amination with suitable transition metals is an efficient and economical way and used frequently on industrial scale and this procedure is successful with noble metal complexes such as $\mathrm{Rh}, \mathrm{Ru}$, and $\mathrm{Pd} / \mathrm{C}$ systems. ${ }^{12,13}$ Homogeneous transition metal catalysts such as $\mathrm{Rh}(\mathrm{I}), \mathrm{Ru}(\mathrm{II})$ and $\operatorname{Ir}(\mathrm{I}-\mathrm{III})^{14-16}$ are also successful tools for the asymmetric reduction of imines/enamines when in complex with different ligands. ${ }^{17}$ These reducing agents are particularly used for an enantioselective synthesis of amines.
Most of the reduction methods are established for the synthesis of primary and secondary amines. However, production of a tertiary amine is more challenging due to the steric demand of two nitrogen-bound organic rests $\left(R_{2}\right.$ and $R_{3}$ in Scheme 2) and the strained formation of the imine/enamine intermediates. Several metal-based catalyst systems (such as $\mathrm{Co}_{3} \mathrm{O}_{4} / \mathrm{H}_{2}$ and $\mathrm{SnCl}_{2} /$ reducing agent) are mentioned in the literature as suitable candidates for this process. ${ }^{\mathbf{1 1 , 1 8 , 1 9}}$ There are also several successful experimental and computational reports on the functionalization of several amines via intra- and intermolecular cyclization reactions in a redox neutral way. ${ }^{20-22}$ In addition, they give some evidence for the formation of ylide intermediates which suggests that the reaction can also proceed via an alternative route and would not necessarily involve the formation of iminium ions. A recent and efficient method is the photocatalytic reduction of iminium ions to produce amines and further functionalize them into diverse drug-like compounds. ${ }^{23}$ Computational approaches can reveal mechanistic pathways and alternatives. ${ }^{24}$ Previous computational studies have focused on the direct reductive amination reaction in terms of transition metal catalysed hydrogenation or the stereoselectivity. ${ }^{25-32}$ The mechanism of enamine formation was investigated using DFT calculations and different functionals. ${ }^{33}$

Processes in chemical industry are undergoing a large change towards the use of feedstock from renewable sources. In a recent study, a catalytic hydroaminomethylation process from sustainable substrate has been introduced to give industrially important surfactant products. ${ }^{6}$

Here, we present a computational study on the thermodynamics and full mechanism of the reductive amination reaction in an organic solvent in the absence and presence of the cocatalyst acid using a combination of an explicit cluster model of solvation and an implicit solvent model to account for long range effects. Information about the thermodynamics, the nonideality of reaction media and the rate-determining step are required for the design of a reaction network model of the reductive amination of sustainable long-chain aldehydes such as tridecanal which can elegantly be generated from a Rh-based hydroformylation reaction of 1-dodecene in a thermomorphic multicomponent solvent system (TMS) ${ }^{34-37}$ or directly in a hydroaminomethylation reaction in a TMS. ${ }^{38}$ 
We here investigate in detail the reductive amination mechanism of propanal by diethylamine to yield $\mathrm{N}, \mathrm{N}$ diethylpropane-1-amine under different reaction conditions:

(a) The reaction in neutral media with an implicit solvent model.

(b) The reaction in the presence of explicitly considered solvent molecules (here water) to form a hydrogen bond network between electrophilic and nucleophilic sites plus an implicit solvation model.

(c) The effect of the co-catalyst acid on the reaction profile.

\section{Methods}

In the scope of this work, a representative reaction between a long chain aldehyde and a secondary amine has been studied in terms of neutral and co-catalyst assisted reaction conditions. In order to simplify the system and reduce the conformational space, propanal was chosen to replace dodecanal $\left(\mathrm{C}_{12} \mathrm{H}_{24} \mathrm{O}\right)$. The calculation of the Gibbs free energy of the reaction shows, however, that the choice of model does not affect the thermodynamics and thus the reaction equilibrium.

The reductive amination process is investigated using $a b$ initio Møller-Plesset perturbation theory (MP). The consideration of electron correlation is crucial to accurately describe the electronic structure of such a system and has to be taken into account which is not fully considered in density functional theory (DFT). The barrier heights of an enamine formation calculated at DFT and the second order Møller-Plesset perturbation theory (MP2) levels were shown to differ by up to $4 \mathrm{kcal} \mathrm{mol}^{-1}$ which is larger than 'chemical accuracy'. In addition, the computed energy barriers in an implicit solvent were found to be lower by $14 \mathrm{kcal} \mathrm{mol}^{-1}$ than in gas phase. ${ }^{33}$ This is also emphasizing the importance of an accurate consideration of solvent effects and the stabilizing effect of explicitly coordinating solvent molecules on the charge distribution and that solvent effects should be taken into account to refine the computed barriers.

All the intermediates and transition state structures were fully optimized by using MP2(full) with a $6-311+\mathrm{g}(\mathrm{d}, \mathrm{p})$ basis set $^{39}$ in Gaussian $09 .^{40}$ Optimized structures were verified by calculation of second derivatives to correspond to minima, whereas a single imaginary frequency characterize a transition state. In order to verify the transition state structures, intrinsic reaction coordinate (IRC) calculations were done along the reaction coordinate by following the gradient in both directions and were found to connect the transition states with the educts and products, respectively. ${ }^{41,42}$ All the energies reported through this work are Gibbs free energies at standard conditions of 298 $\mathrm{K}$ and 1 bar. Thermodynamic corrections were taken from MP2 frequency calculations at $298 \mathrm{~K}$ and added to the single point SMD MP2 energies. Full energies plus thermodynamic corrections of all species are given in the ESI. $\dagger$ Charge analysis on the optimized structures was performed using the Natural Bond Orbital (NBO) method. ${ }^{43,44}$ The bond distances are given in units of $\AA$ written in black and NBO charges in blue where appropriate.
Single point implicit solvent calculations were carried out at the optimized geometries with the same level of theory in order to estimate the effect of the solvent on the reaction profile. The solvent effects were included using the solvation model based on density (SMD) method for $N, N$-dimethylformamide (DMF) as a polar and decane as an apolar solvent to mimic the polarity effect ( $\varepsilon=36.7$ and 2 , respectively).$^{45}$ The choice of solvents correspond to that used in the experiments for the hydroformylation reaction of dodecene. ${ }^{35,36}$ In the main text of this manuscript, we only report Gibbs free energies in the presence of DMF and decane. Results of calculations in the gas phase can be found in the ESI $\dagger$ for all reaction pathways. Generally, polar and apolar solvents have a strong effect on the thermodynamics and kinetics of the reductive amination reaction. Since we are focusing on the control by choice of solvent, only the results in solution are given in the main text.

\section{Results and discussion}

\subsection{Reaction thermodynamics}

The thermodynamics of a reaction are critical parameters for simulations of chemical reaction networks ${ }^{46}$ and entire processes. ${ }^{47}$ Standard thermodynamic parameters such as reaction enthalpy and Gibbs free energy of the ideal system are often not available in the literature but can be obtained computationally and then later combined with other thermodynamic approaches to account for the non-ideality of complex reaction mixtures at process conditions..$^{35}$ Solvent effects on the kinetics ${ }^{48}$ and thermodynamics ${ }^{49}$ of the hydroformylation reaction of 1-dodecene were already investigated experimentally and combined with the Perturbed Chain Statistical Associating Fluid Theory (PC-SAFT) to model the reaction of dodecene with syngas $\left(\mathrm{CO} / \mathrm{H}_{2}\right)$ in a solvent mixture of DMF/decane at $90^{\circ} \mathrm{C}$ and 21 bar. Quantum chemical calculations of thermochemical data pose a challenge in terms of accuracy and standard DFT functionals were not suitable to obtain accurate data for the hydroformylation of long chain olefins but MP2 calculations gave reliable thermodynamic equilibrium constants $K_{\mathrm{f}}{ }^{49}$

The reaction thermodynamics $\Delta G_{\mathrm{r}}^{\circ}$ of the reductive amination reaction of aldehydes of various carbon chain lengths and diethylamine were calculated in the gas phase and in an implicit solvent environment. The free energy of the reaction was $-14.7 \mathrm{kcal} \mathrm{mol}^{-1}$ and only slightly affected by presence of a solvent to become -15.1 and $-15.3 \mathrm{kcal} \mathrm{mol}^{-1}$ in either DMF and decane (see Table 1).

Table 1 The thermodynamics $\left(\Delta G_{\mathrm{r}}^{\circ}, \mathrm{kcal} \mathrm{mol}^{-1}\right)$ and solvent effects of the reductive amination reaction of various aldehydes with diethylamine $\left(\left(\mathrm{C}_{2} \mathrm{H}_{5}\right)_{2} \mathrm{NH}\right)$

\begin{tabular}{llll}
\hline & \multicolumn{2}{l}{ Free energy of reaction $\left(\Delta G_{\mathrm{r}}^{\circ}, \mathrm{kcal} \mathrm{mol}^{-1}\right)$} \\
\cline { 2 - 4 } Aldehyde & Gas phase & DMF & Decane \\
\hline $\mathrm{C}_{3} \mathrm{H}_{6} \mathrm{O}$ & -14.7 & -15.1 & -15.3 \\
$\mathrm{C}_{5} \mathrm{H}_{10} \mathrm{O}$ & -14.5 & -15.4 & -15.2 \\
$\mathrm{C}_{9} \mathrm{H}_{18} \mathrm{O}$ & -14.5 & -15.3 & -15.1 \\
$\mathrm{C}_{10} \mathrm{H}_{20} \mathrm{O}$ & -14.4 & -15.3 & -15.1 \\
$\mathrm{C}_{11} \mathrm{H}_{22} \mathrm{O}$ & -14.3 & -15.2 & -15.0
\end{tabular}



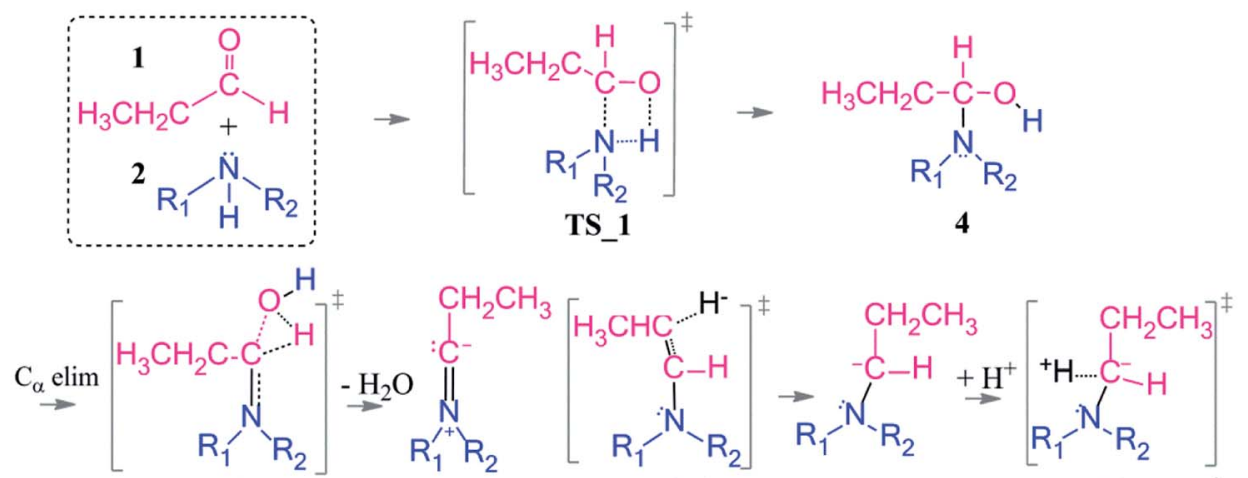

TS_2a

$5 a$

TS_3

6

TS_5
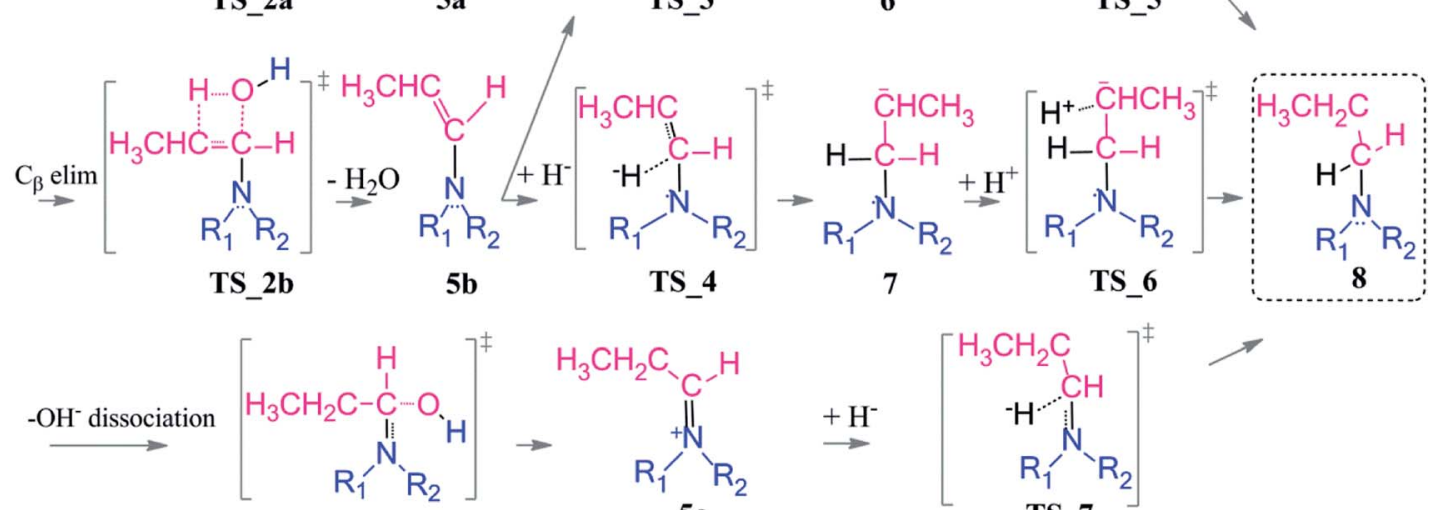

TS_7

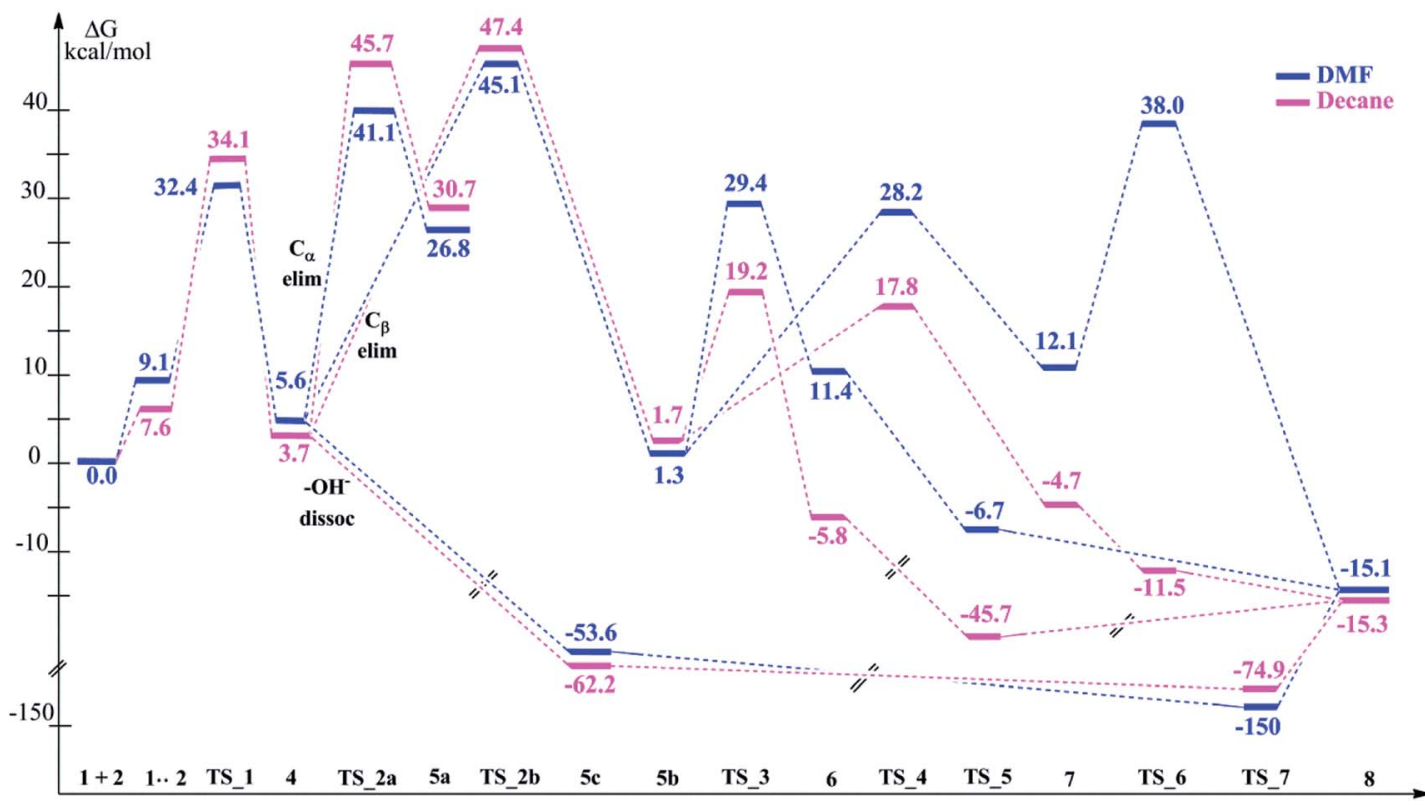

Scheme 3 Reaction mechanism including transition states and intermediate structures (top) and the free energy profile for reductive amination reaction in neutral media (bottom), $\left(\mathrm{R}_{1}=\mathrm{R}_{2}=-\mathrm{CH}_{2} \mathrm{CH}_{3}\right)$.

Then, the effect of the chain length of the aldehyde on the thermodynamics of the reductive amination reaction was investigated. The free energy of the reaction is almost independent of the chain length and between -14.3 and $-14.7 \mathrm{kcal} \mathrm{mol}^{-1}$ for all carbon chain lengths, and the effect of the solvent is only minor.
One can thus conclude that the thermodynamics of the reaction is independent from the chain length of the aldehyde and a truncated substrate model is appropriate. Thus, we investigated the mechanism of the reductive amination only for the short chain aldehyde, e.g. for the reaction of propanal and diethylamine. 


\subsection{Reaction mechanism}

Reductive amination of an aldehyde starts with a nucleophilic addition of the amine to the carbonyl group of the aldehyde. The initial step of the reaction leads to the formation of a carbinolamine intermediate. In an intramolecular condensation reaction, an enamine or an iminium ion are formed depending on the chosen reaction conditions (in particular the $\mathrm{pH}$ of the reaction medium). This enamine formation is an equilibrium reaction and can be controlled by $\mathrm{H}_{2} \mathrm{O}$ addition or removal in a chemical process. At the final step, the product amine is generated by reduction of the enamine or iminium. Throughout the reaction process, there are several key structures and the reaction is proceeding via multiple transformations in order to form the tertiary amine as a final product. These chemical transformations are controlled by several factors like the $\mathrm{pH}$ of the reaction medium (see above), the polarity of the solvent, explicit formation of hydrogen bonds by coordinating polar reaction media, and activation of the intermediates by Lewis base/Brønsted acid addition. During an automated optimization the amine formation during reductive amination takes around 2 minutes with moderate to high yields (67-97\%). The conversion rate is lowered by the steric amines and results in byproduct formation via simple reduction. ${ }^{\mathbf{5 0}}$

3.2.1 The reaction in neutral media. In neutral media, the aldehyde (1) and the amine (2) form a pre-complex before the nucleophilic addition of the lone pair of the nitrogen to the positively polarized carbon atom of the aldehyde occurs (Scheme 3) in a barrierless process. This pre-complex is an intermediate and 9.1-7.6 $\mathrm{kcal} \mathrm{mol}^{-1}$ higher in energy than the substrates. Patil and Sunoj also report a barrierless nucleophilic attack from DFT mPW1PW91 calculation and an increase by $5 \mathrm{kcal} \mathrm{mol}{ }^{-1}$ upon pre-complex formation. ${ }^{33} \mathrm{~A}$ hemiaminal intermediate (4) is formed via an intramolecular proton transfer from the amine to the carbonyl oxygen in a concerted fashion (TS_1) with an activation barrier of $33.3 \mathrm{kcal} \mathrm{mol}^{-1}$ (see ESI, Table S1 $\dagger$ ) in the gas phase and $32.4-34.1 \mathrm{kcal} \mathrm{mol}^{-1}$ in DMF and decane, respectively, which agree with the $38 \mathrm{kcal} \mathrm{mol}^{-1}$ in the gas phase $\left(24 \mathrm{kcal} \mathrm{mol}^{-1}\right.$ in THF). ${ }^{33}$ The polar solvent DMF only slightly stabilizes the transition state. A co-crystal structure of a hemiaminal and diethylamine complex gives an increase in bond length of $\mathrm{C}-\mathrm{OH}$ from 1.410 to $1.430 \AA$ Ahich is in excellent agreement with the calculated $1.420 \AA$ for our hemiaminal structure (4). ${ }^{51}$ Moreover, the position and the interactions of the hydrogen atoms in the co-crystal structure were suggested to be an indicator of a concerted mechanism which was also observed in the computations (TS_1).

The first coupling step is followed by an intramolecular condensation reaction and release of a water molecule, formed by the $-\mathrm{OH}$ group with a proton from the hemiaminal (4), see Scheme 3.

In the condensation step, a proton from either an $\alpha$ - or a $\beta$ carbon atom can be eliminated. Abstraction of the proton at $\alpha$ position via TS_2a leads to formation of the 5a intermediate whereas when the $\beta$-proton is eliminated via $\mathbf{T S} \_2 \mathbf{b}$ and the enamine intermediate $\mathbf{5 b}$ is formed. The activation barriers for both routes are $48.9 \mathrm{kcal} \mathrm{mol}^{-1}$ in the gas phase. They are lowered by 5-10 kcal mol ${ }^{-1}$ in a solvent (but still larger than $40 \mathrm{kcal} \mathrm{mol}^{-1}$ in both DMF and decane solvents) with the $\alpha$ pathway being slightly favoured. This is significantly lower than the $58-60 \mathrm{kcal} \mathrm{mol}^{-1}$ reported in the literature. ${ }^{33}$ The resulting intermediates $\mathbf{5 a}$ and $\mathbf{5 b}$ (enamine) are very different in stability (Scheme 3, bottom). The energy difference of $\mathbf{5 a}$ by 25$29 \mathrm{kcal} \mathrm{mol}^{-1}$ compared to $\mathbf{5 b}$ can be attributed to the larger charge separation in the structure and the re-hybridization character of the $\mathrm{C}_{\alpha}$. Whereas in $\mathbf{5 b}$, the newly formed double bond has a planar geometry with a trans orientation of the alkyl groups and a charge density that is evenly distributed along the structure. These are the factors which are stabilizing $\mathbf{5 b}$ with respect to 5a (see Fig. 1).

After examining the elimination from $\alpha$ and $\beta$ positions, we are also considering the possibility of the dissociation of the hydroxyl anion without abstracting any proton (Scheme 3 ). This dissociation can occur by assistance of the solvent or the counter cations in the reaction medium. As a result of this dissociation, an iminium intermediate (5c) can be generated. This commonly accepted intermediate is formed in a highly exergonic fashion (around -54 to $-63 \mathrm{kcal} \mathrm{mol}^{-1}$ ). The elongation of the $\mathrm{C}-\mathrm{OH}$ bond takes place easily and without any barrier to release $\mathrm{OH}^{-}$and results a stable iminium cation.

The final step in the reductive amination is the hydrogenation (reduction) of intermediates $\mathbf{5 a}, \mathbf{5 b}$ and $\mathbf{5 c}$ to generate the desired tertiary amine. Besides the conventional organic boro

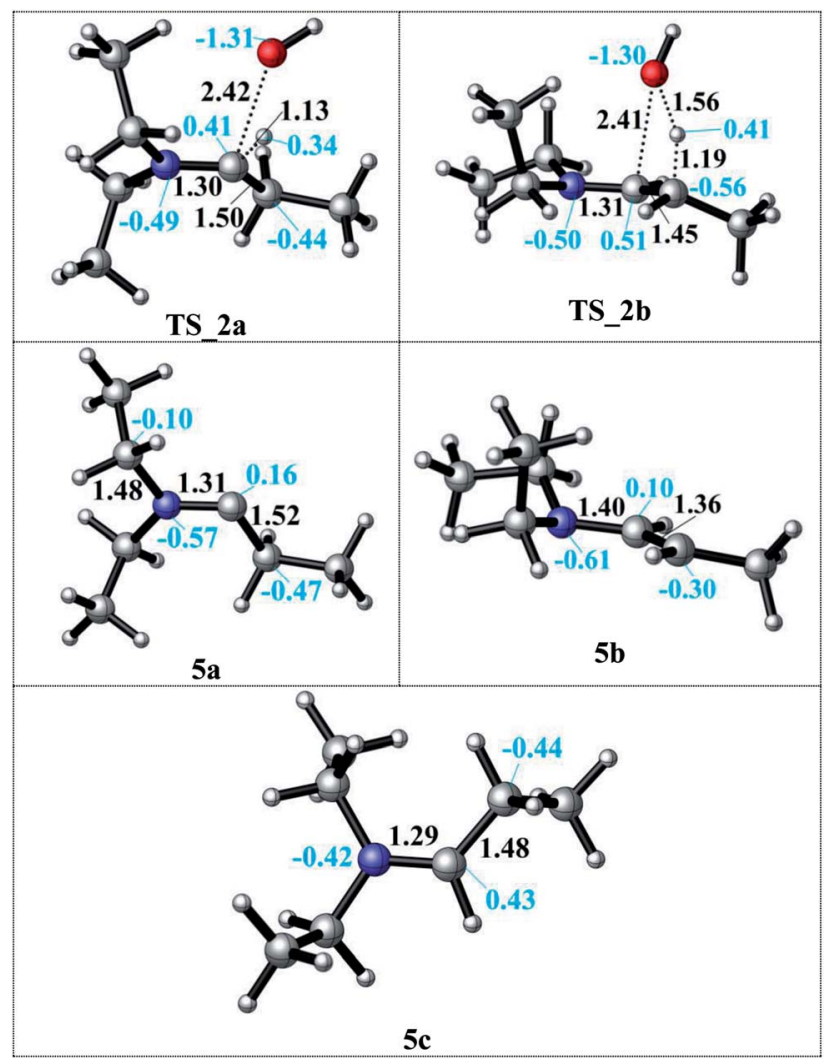

Fig. 1 Details of the formation of the $5 a$ and $5 b$ intermediates after proton abstraction to release water via $\mathrm{TS} \_2 \mathrm{a}\left(\mathrm{C}_{\alpha}\right.$. position) and via TS_2b ( $C_{\beta}$ position) and the formation of the iminium ion $5 c$ upon $\mathrm{OH}^{-}$ dissociation. 


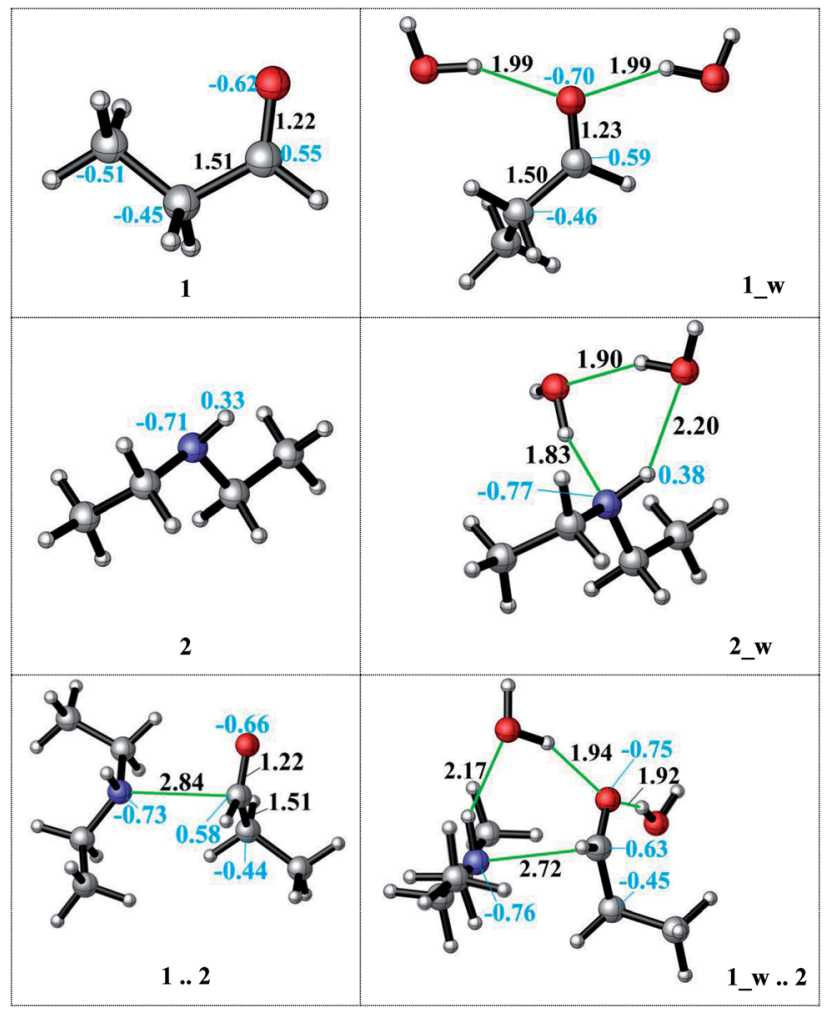

Fig. 2 The effect of the water assistance on the pre-complex formation. (distances are given in $\AA$, black; NBO charges are presented in blue).

hydride derivatives, there are several novel approaches for a mild and selective reduction and most of them consider a transition metal (mostly $\mathrm{Ru}$ and $\mathrm{Rh}$ ) catalyzed hydrogenation. ${ }^{52} \mathrm{~A}$ kinetic study suggests that the rate of the reaction is independent of the imine concentration and organoborane catalyzed reduction is the rate determining step..$^{53}$ In this work, we investigate a sequential approach for hydrogenation since that reflects also the procedure when an external hydride donor or an activated $\mathrm{H}_{2}$ molecule bound to a transition metal catalyst is used. We did not consider the hydride donor or the catalyst in molecular details since this is highly dependent on each experimental set-up and the choice of the reducing agent.

Since the enamine $\mathbf{5 b}$ is $25-29 \mathrm{kcal} \mathrm{mol}^{-1}$ lower in energy than $\mathbf{5 a}$, only reduction of the enamine was investigated by addition of first the hydride $\left(\mathrm{H}^{-}\right)$and then the proton $\left(\mathrm{H}^{+}\right)$ species. The hydride addition to the enamine $\mathbf{5 b}$ structure was investigated for both carbon atoms of the $\mathrm{C}=\mathrm{C}$ double bond $\left(\mathrm{C}_{\beta}\right.$ via $\mathbf{T S} \_3$ to give $\mathbf{6}$ and $\mathrm{C}_{\alpha}$ via $\mathbf{T S} \_\mathbf{4}$ to give 7 ). The $\mathrm{C}_{\alpha}$ atom in $\mathbf{5 b}$ has a charge of 0.10 , where $\mathrm{C}_{\beta}$ is negatively charged (Fig. 1). Thus, the attack of the $\mathrm{H}^{-}$to the positively charged $\mathrm{C}_{\alpha}$ carbon via TS_4 to generate structure 7 is found to be kinetically favourable with a difference in transition state barriers of 1.2$1.4 \mathrm{kcal} \mathrm{mol}^{-1}$ but thermodynamically less preferred by $0.7-$ $1.1 \mathrm{kcal} \mathrm{mol}^{-1}$. Thus, we consider the presence of both intermediates 6 and 7 in the protonation step to give the final product 8. Intermediate 6 has a higher proton affinity by $44-$ $47 \mathrm{kcal} \mathrm{mol}^{-1}$ than 7 and consequently the proton addition via TS_5 is thermodynamically preferred. TS_5 was found to be lower in energy than TS_6 in decane but upshifted by around $40 \mathrm{kcal} \mathrm{mol}^{-1}$ in DMF due to the solvent shielding of electrostatics. This destabilization leads to a barrier of around $26 \mathrm{kcal} \mathrm{mol}^{-1}$ for TS_6 in DMF which was exothermic in case of TS_5. The large destabilizing effects for the transition states TS_5 and TS_6 in polar media such as DMF indicate the need for a careful design of a suitable solvent to thermodynamically and kinetically enable the reaction.

The reduction of the intermediate $\mathbf{5 c}$ is quite distinct from the one of $\mathbf{5 b}$. Due to the positive charge on the iminium ion, only hydride addition is sufficient to obtain the final product amine. Here, hydride addition occurs exergonically by -75 to $-150 \mathrm{kcal} \mathrm{mol}^{-1}$. It is also noteworthy to mention that the polar solvent DMF stabilizes the charged transition state TS_7 and highly favors the hydride addition. As a result, reduction of $\mathbf{5 c}$ is preferred over that of $\mathbf{5 b}$.

In conclusion, once the hemiaminal (4) is formed via the coupling reaction, transformation to the final product is thermodynamically favorable. This transformation will preferably take place via the iminium intermediate $(\mathbf{5 c})$ which is more stable than $\mathbf{5 b}$ and once again due to highly exergonic single step reduction to give the long chain amine.

3.2.2 Explicit water-coordination. Water is the prime green solvent of a chemical reaction and was also shown to accelerate the reductive amination reaction itself. ${ }^{14}$ It is also observed that $\mathrm{H}_{2} \mathrm{O}$ addition has a positive influence to avoid byproduct formation, catalyst poisoning, and also to promote the reduction. ${ }^{13,54}$ At the same time, the presence of an excess of water might lead to the unwanted backward reaction and hydrolysis of the enamine intermediate.

The direct reductive amination reaction can be catalysed by non-aqueous hydrogen bonding solvents e.g. via thiourea coordination by means of the imine activation in the absence of an acid. ${ }^{55}$ Moreover, kinetic studies show that the added amount of the H-bond donor has no effect on the ratedetermining step and is only needed in catalytic amounts. Additionally, the role of a hydrogen bond network has been shown to facilitate the nucleophilic addition and stabilize intermediates. ${ }^{33,56} \mathrm{~A}$ diethylamine and an acid complex was crystallized as an intermediate structure during a reductive amination which highlights the importance of the H-bond interactions during the reaction..$^{51}$ We here investigate the catalytic effect and mechanistic role of explicitly coordinating water molecules on the kinetics (transition state barriers) and thermodynamics in the reductive amination. Several possible coordination modes of the water molecules via $\mathrm{H}$-bonding were analysed to model the reaction medium. Initially, water coordination to the amine and to the aldehyde was established individually. First, we hypothesized that the H-bond stabilization of the amine is more likely due to its basic nature. The assistance of two explicit water molecules (complex 2_w, see Fig. 2) provides $9-4 \mathrm{kcal} \mathrm{mol}^{-1}$ extra stabilization to the amine in DMF and decane, respectively. The charge on the nitrogen atom changes (from -0.71 to -0.77 ) and water coordination thus increases the nucleophilicity of the nitrogen atom.

Then, solvation of propanal by two assisting water molecules which form hydrogen bonds with the oxygen lone pairs was 


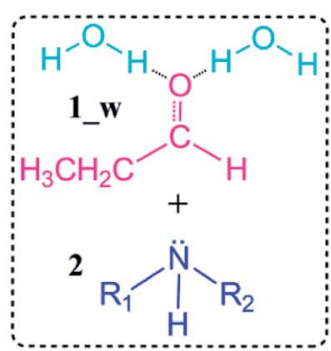<smiles>[R2]N([R2])[C@@H](CC)[C@H](O[Hg])[Hg]O[3H]</smiles><smiles>[R2]N([R2])[C@@H](CC)[C@@H](O[Hg])[C@H](C)O</smiles>

1w_2<smiles>[R2]N([R2])[C@]([2H])(CC)C(O)O</smiles>

TS_1_w
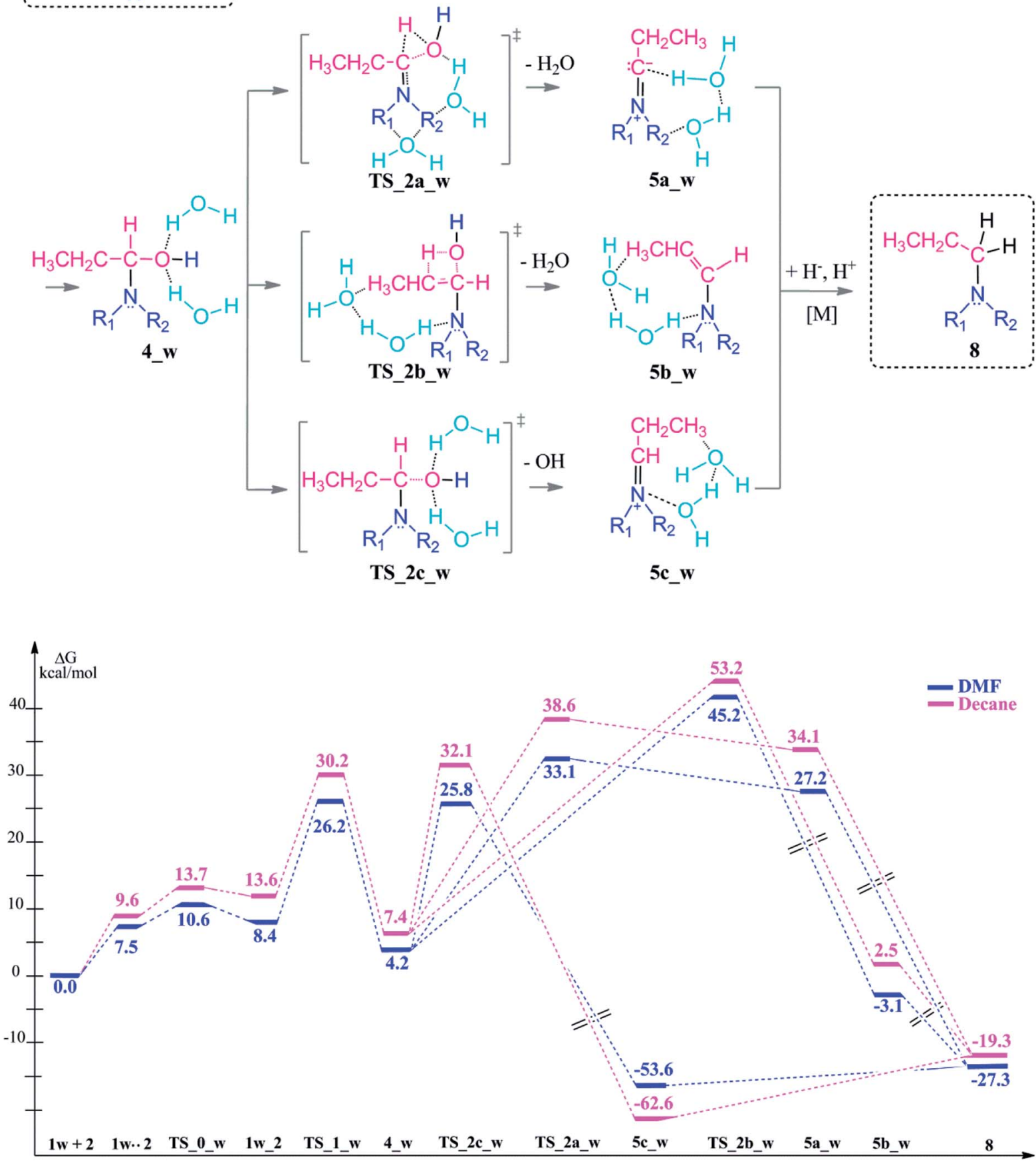

Scheme 4 Reaction mechanism including transition states and intermediates and the free energy profile for reductive amination reaction with explicit water assistance, $\left(\mathrm{R}_{1}=\mathrm{R}_{2}=-\mathrm{CH}_{2} \mathrm{CH}_{3}\right)$.

investigated. This H-bonding stabilizes the initial aldehyde by 12-4 kcal mol ${ }^{-1}$ in DMF and decane, respectively. The charge of the reactive carbon atom changes from 0.55 to 0.59 by explicit water coordination, and thus the electrophilicity was promoted. After observing the favorable effect of the explicit H-bonding, we considered a full first solvation shell to connect the amine and 

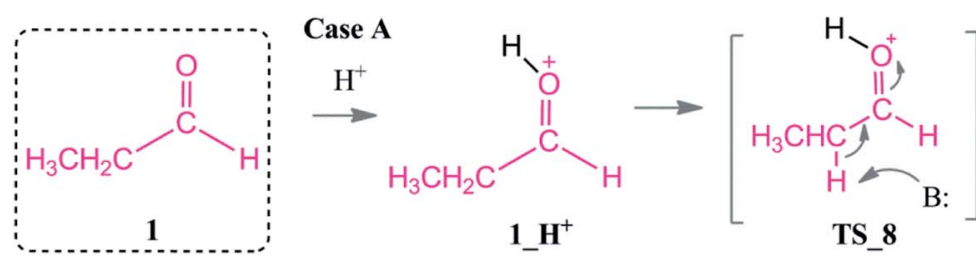<smiles>CC=CO</smiles>
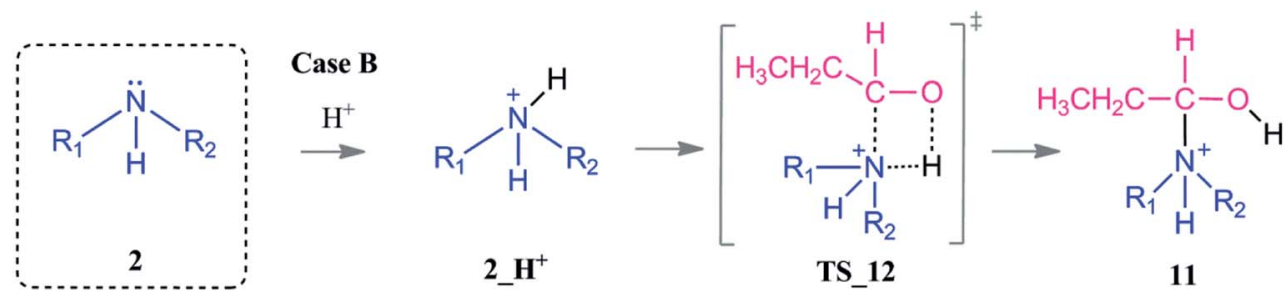

Scheme 5 Coordination modes of an acid which acts as a co-catalyst via direct protonation of the aldehyde (Case A) and amine (Case B).

the aldehyde by a detailed hydrogen bonding network of the water molecules. In our situation, however, such a watermediated hydrogen bonding was not found to be feasible due to the hydrophobic character of the long carbon chains. Moreover, if a hydrogen-bonded cage-like structure was to be identified in quantum chemical calculations, it would be too minor and not be stable and persistent at experimental reaction conditions above a temperature of $100{ }^{\circ} \mathrm{C}$.

Although the water coordination increases the nucleophilicity of the amine, this coordination hinders the nucleophilic attack and a desolvation has to occur first. In case of aldehyde solvation, the water coordination has a positive effect on the charge density. One of the coordinating water molecules can occupy a bridging position between the amine (H-bond donor) and the aldehyde (H-bond acceptor) and facilitate the attack (Fig. 2,1_w..2). Thus, the resulting aldehyde complex (1_w) was used as the starting structure (see Scheme 4) during the explicit solvent-assisted reaction. Subsequently, the entire reaction pathway including transition state localizations was elucidated in detail.

Upon hydrogen bonding, the electrophilicity of the carbonyl carbon atom in 1_w structure increases due to the increase in charge on the oxygen atom (from -0.62 to -0.70 , see Fig. 2, top). In the aldehyde-amine pre-complex, this leads to an extra stabilization by $10.6-6 \mathrm{kcal} \mathrm{mol}^{-1}$ in DMF and decane due to the water molecule mediated interaction between aldehyde and amine (see Fig. 2, bottom). The pre-complex 1_w .. 2 is more tightly bound and the $\mathrm{N} \cdots \mathrm{C}$ distance decreases from $2.84 \AA$ to $2.72 \AA$. Coordination by water molecules leads to a different stepwise nucleophile attack pathway which now proceeds via TS_0_w (amine addition) to give 1w_2 and via $\mathbf{T S} \_\mathbf{1} \_\mathbf{w}$ (proton transfer) to 4_w (see Scheme 4, top). Here, the nucleophilic addition proceeds in a stepwise fashion whereas in the absence of water it proceeded in a concerted fashion (TS_1, Scheme 3). Explicit water coordination lowers the barrier of activation by 5.5-9.9 $\mathrm{kcal} \mathrm{mol}^{-1}$ which is in agreement with similar results obtained for one and two methanol molecules using DFT calculations. ${ }^{33}$ Also for the Schiff base formation, a decrease of energy barriers by inclusion of explicit waters was reported..$^{57,58}$
After the formation of $\mathbf{4}$-w, the water elimination step by proton abstraction from the $\mathrm{C}_{\alpha}$ carbon is facilitated by explicit water coordination. This solvent cage lowers the barrier of the condensation reaction, particularly for the transition state TS_2a_w (by 8-7.1 $\mathrm{kcal} \mathrm{mol}^{-1}$ ) where one solvent water coordinates to the leaving water molecule. On the other hand, water coordination disfavours elimination from $\mathrm{C}_{\beta}$ of the enamine TS_2b_w by $0.1-5.8 \mathrm{kcal} \mathrm{mol}^{-1}$.

The dissociation of the $-\mathrm{OH}$ group by the assistance of the two water molecules (TS_2c_w) has an energy barrier of only 25.8-32.1 kcal mol ${ }^{-1}$ which is the lowest energy TS of all elimination possibilities. This reaction step is exergonic by $53.6-$ $62.6 \mathrm{kcal} \mathrm{mol}^{-1}$ and results an iminium ion complex (5c_w) with two coordinating water molecules.

The introduction of the explicit solvation to the system effects also the energy barriers for the following water release during the condensation reaction. When solvent molecules are considered, water release via $\mathrm{C}_{\alpha}$ proton abstraction is kinetically favored over $\mathbf{C}_{\beta}$ (TS_2a_w vs. TS_2b_w). However, the relative thermodynamic stabilities of the intermediates $\mathbf{5 a}, \mathbf{5 b}, \mathbf{5 c} v s$. 5a_w, 5b_w, 5c_w are preserved in neutral and water coordinated systems and in favor of the $\mathbf{5 c}$ and $\mathbf{5 c} \_\mathbf{w}$ formation.

We can thus show that the choice of an explicitly coordinating hydrogen-bonding solvent (here water) significantly changes the kinetic control of the reductive amination in that initial coupling step although it does not completely change the prefererred reaction pathway.

3.2.3 The role of an acid as a co-catalyst. Experimentally, the presence of an acid as a co-catalyst was found to favorably influence the reductive amination reaction. For example, an optimum pH range of 2.5-3.5 in aqueous solution gives the best performance for cyclometallated iridium complexes in a formic acid/formate buffer solution. ${ }^{14}$ Different aspects of the role of the acid addition in the reductive amination reaction are discussed. Some suggest that the presence of a weak acid will facilitate and promote the first addition step whereas strongly acidic solutions would prevent further reactions of the initially formed primary amine by forming the ammonium salt. ${ }^{13}$ The $\mathrm{pH}$ value is particularly relevant for an optimization of the catalytic selectivity and the yield of the reaction. It was also 


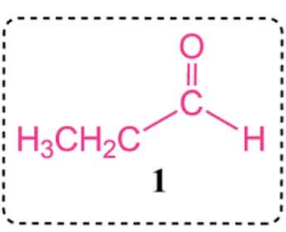<smiles>C[InH2]</smiles>

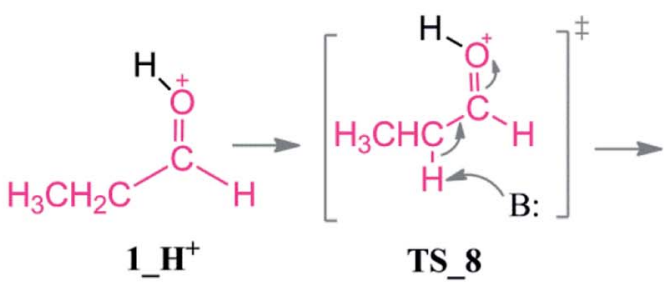

TS_8

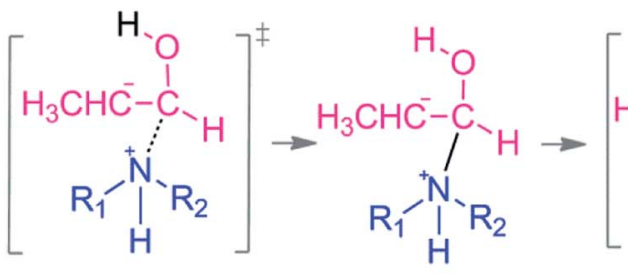

TS_10
9<smiles>[R2][NH+]([R2])[C@@H](O)[CH]C</smiles>

TS_11<smiles>[R2][NH+]([R7])/C=C(/C)O</smiles>

10

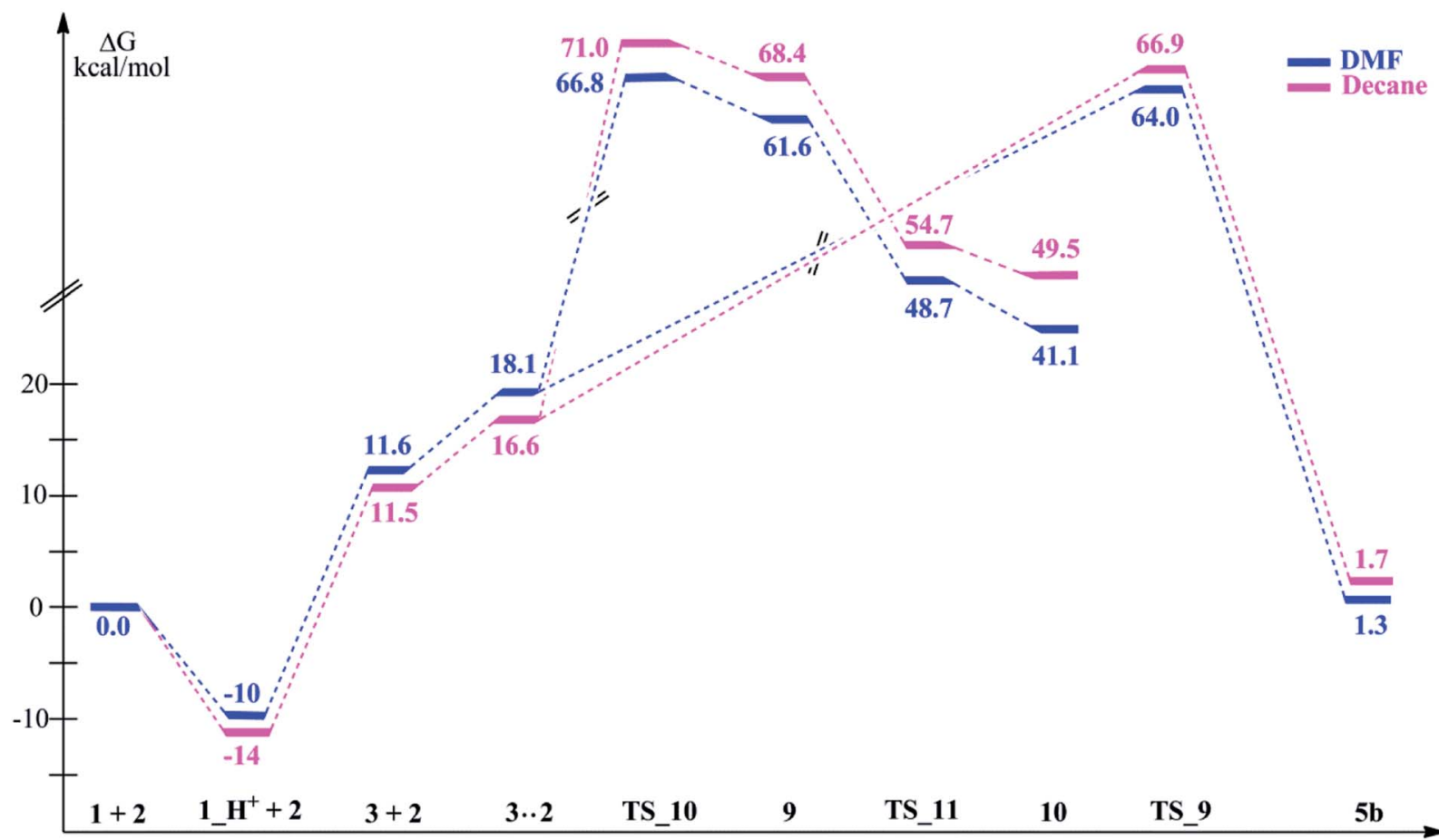

Scheme 6 Reaction mechanism including identified transition states and intermediate structures and the free energy profile for reductive amination reaction following the keto-enol tautomerization, $\left(\mathrm{R}_{1}=\mathrm{R}_{2}=-\mathrm{CH}_{2} \mathrm{CH}_{3}\right)$.

shown that the $\mathrm{pH}$ level of the reaction media can affect the catalyst activity, its enantioselectivity and in particular the kinetics by a factor of six. ${ }^{\mathbf{1 4}}$

We here investigate the role of an acid which acts as a cocatalyst in the reductive amination reaction at mildly acidic conditions ( $\mathrm{pH} 4$ or 5 ; for example acetic acid or formic acid). In order to rationalize the role of the acid as a co-catalysts, two scenarios were investigated.

In the presence of an acid, protonation of both the aldehyde and amine are possible (Scheme 5). As a first case scenario, protonation of the aldehyde was investigated (Case A). Here, the carbonyl oxygen of the aldehyde is protonated $\left(\mathbf{1} \_\mathbf{H}^{+}\right)$and afterwards an alpha proton is abstracted (TS_8) by the amine (which was also considered as the base here) with a barrier of 9$3 \mathrm{kcal} \mathrm{mol}^{-1}$. As a result of this keto-enol tautomerization, the aldehyde is in equilibrium with its enol structure. The keto-enol equilibrium is known to be thermodynamically driven and usually favorable on the keto side. Thus, the aldehyde (1) is lower in energy by around $12 \mathrm{kcal} \mathrm{mol}^{-1}$ than the enol compound (3).

The protonation of the amine is examined as Case $\mathrm{B}$. This step is feasible due to the basic character of the amine. In a recent study on reductive amination reaction with $\mathrm{Rh}$ complexes, it is suggested that this protonation of amine plays 


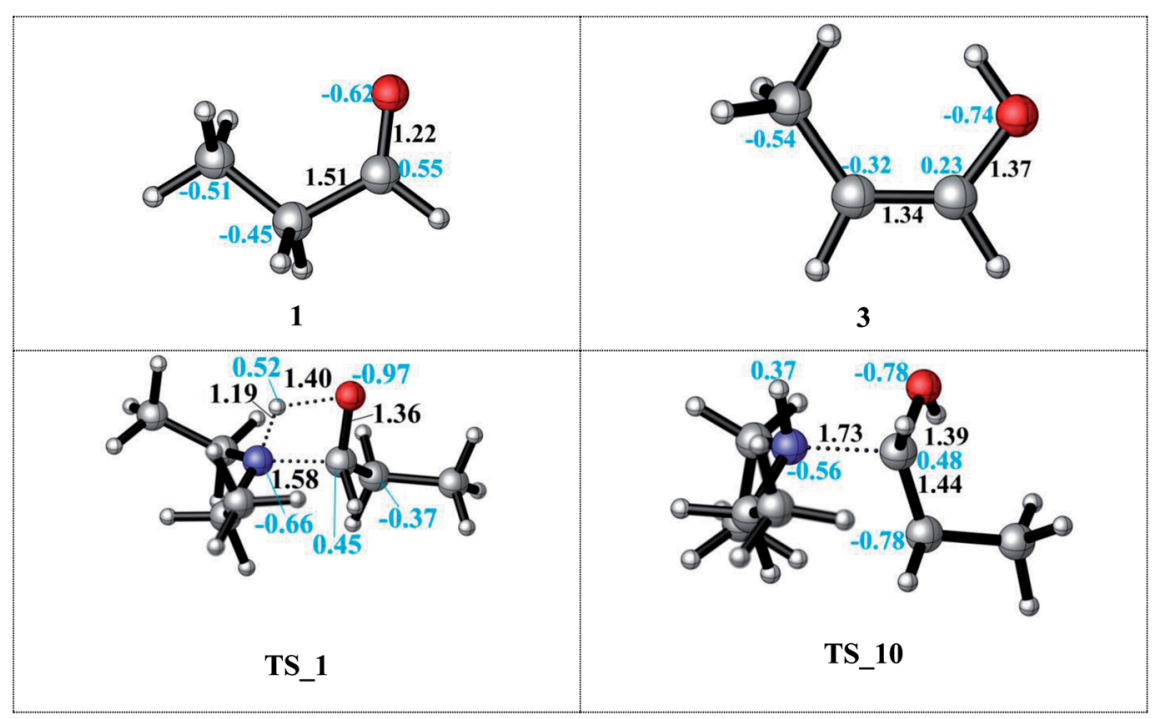

Fig. 3 Details of the role of the acid co-catalyst in the nucleophilic attack of the amine. (Left) aldehyde substrate and TS_1 in neutral media. (Right) enol substrate and TS_10 in acidic media.

a key role in the catalytic cycle especially in the absence of an external hydrogen source. ${ }^{59}$ However, the nucleophilicity of the amine is abandoned by the formation of a quaternary ammonium compound $\left(\mathbf{2} \mathbf{H}^{+}\right)$which will disable the attack to the aldehyde. In this respect, we identified an intermolecular proton transfer from the ammonium compound to the carbonyl oxygen of the aldehyde. We successfully located the transition state (TS_12) for a concerted proton transfer and nucleophilic addition to the aldehyde. The proton affinity of the amine is around $43 \mathrm{kcal} \mathrm{mol}^{-1}$ higher than that of the aldehyde and thus the protonation of the amine (Case B) is thermodynamically more favorable.

However, besides the favorable thermodynamics upon protonation, the kinetic feasibility of the reaction after protonation of the reactants is still a question. First, we are investigating the reaction pathway following the enol formation. Then, the mechanism of the reaction via initially protonated structures (Case A vs. Case B) will be discussed in comparison.

3.2.4 Reaction path following the keto-enol tautomerization. Protonation of the aldehyde $\left(\mathbf{1}_{-} \mathbf{H}^{+}\right)$is exothermic by $10-$ $14 \mathrm{kcal} \mathrm{mol}^{-1}$. After the keto-enol tautomerization (see above), the reaction between the enol and the substrate diethylamine was investigated (Scheme 6). Two different pathways which both lead to the enamine intermediate can be distinguished. The first route is following the nucleophilic attack via TS_10 $\left(>66 \mathrm{kcal} \mathrm{mol}^{-1}\right.$ ) which then leads to the high energy adduct 9 which is $62-68 \mathrm{kcal} \mathrm{mol}^{-1}$ higher in energy than the substrates. From $9, \mathrm{C}_{\alpha}$ proton abstraction and water elimination may proceed. Here, the leaving $-\mathrm{OH}$ group is stabilized by intramolecular interactions with the nearby methyl hydrogen atoms of the alkyl chain. Then, elimination from the alpha position occurred via TS_11 to give the enamine zwitter ion $\mathbf{1 0}$ which is high in energy and inaccessible.

The second route is the nucleophilic addition of the amine and an intramolecular water elimination by abstraction of a proton from the positively charged nitrogen atom via TS_9. This transition state barrier is comparable to TS_10 (within 3$4 \mathrm{kcal} \mathrm{mol}^{-1}$ ) but the formation of the intermediate $\mathbf{5 b}$ is thermodynamically more favorable and it is significantly more stable compared to 10 by $40-48 \mathrm{kcal} \mathrm{mol}^{-1}$. After the formation of $5 \mathbf{b}$, hydrogenation proceeds in analogy to the neutral pathway (Scheme 3) by first hydride binding to $\mathrm{C}_{\alpha}$ or $\mathrm{C}_{\beta}$ and subsequent protonation of the remaining carbon atom.

Protonation of the aldehyde and then formation of the enol significantly affects the charge distribution in the substrates (see Fig. 3). Protonation of the oxygen atom decreases the electrophilicity of the carbon atom from an atomic charge of 0.55 for the aldehyde to 0.23 for the enol. Thus, the reactivity of the carbon center is lowered and renders this reaction pathway inaccessible. This effect is also reflected in the activation barriers of this step (TS_10 vs. TS_1) which show a difference of $32.4-36.9 \mathrm{kcal} \mathrm{mol}^{-1}$ and an elongation of the $\mathrm{N} \cdots \mathrm{C}$ distance from 1.58 to $1.73 \AA$ in the transition state (see Fig. 3).

3.2.5 Initial protonation of the substrates (Case A vs. Case B). After initial protonation of the aldehyde $\left(\mathbf{1} \_\mathbf{H}^{+}\right)$mentioned as Case A, amine attack is also considered without enol formation taking place (Scheme 7). In this case, the protonated aldehyde and amine are approaching each other due to the enhanced electrophilicity of the carbonyl group. A pre-complex formation is exergonic by 36 to $42 \mathrm{kcal} \mathrm{mol}^{-1}$ and the following nucleophilic addition takes place via TS_14 with a small barrier of $3 \mathrm{kcal} \mathrm{mol}^{-1}$ only. The resulting intermediate $\mathbf{1 1}$ is around $10 \mathrm{kcal} \mathrm{mol}^{-1}$ lower than the transition state.

In TS_13, ${ }^{-} \mathrm{OH}$ and a proton from the nitrogen are the leaving groups. Here, $\alpha$ and $\beta$ eliminations are not feasible because of the acidic character of the ammonium proton. Thus, water elimination easily proceeds via $\mathbf{T S} \_\mathbf{1 3}$ as the only distinct way. As a result of this elimination, the iminium intermediate $\mathbf{5 c}$ is obtained as the only candidate. Thus, the final reduction of intermediate $\mathbf{5 c}$ (via the same reduction procedure as applied in 


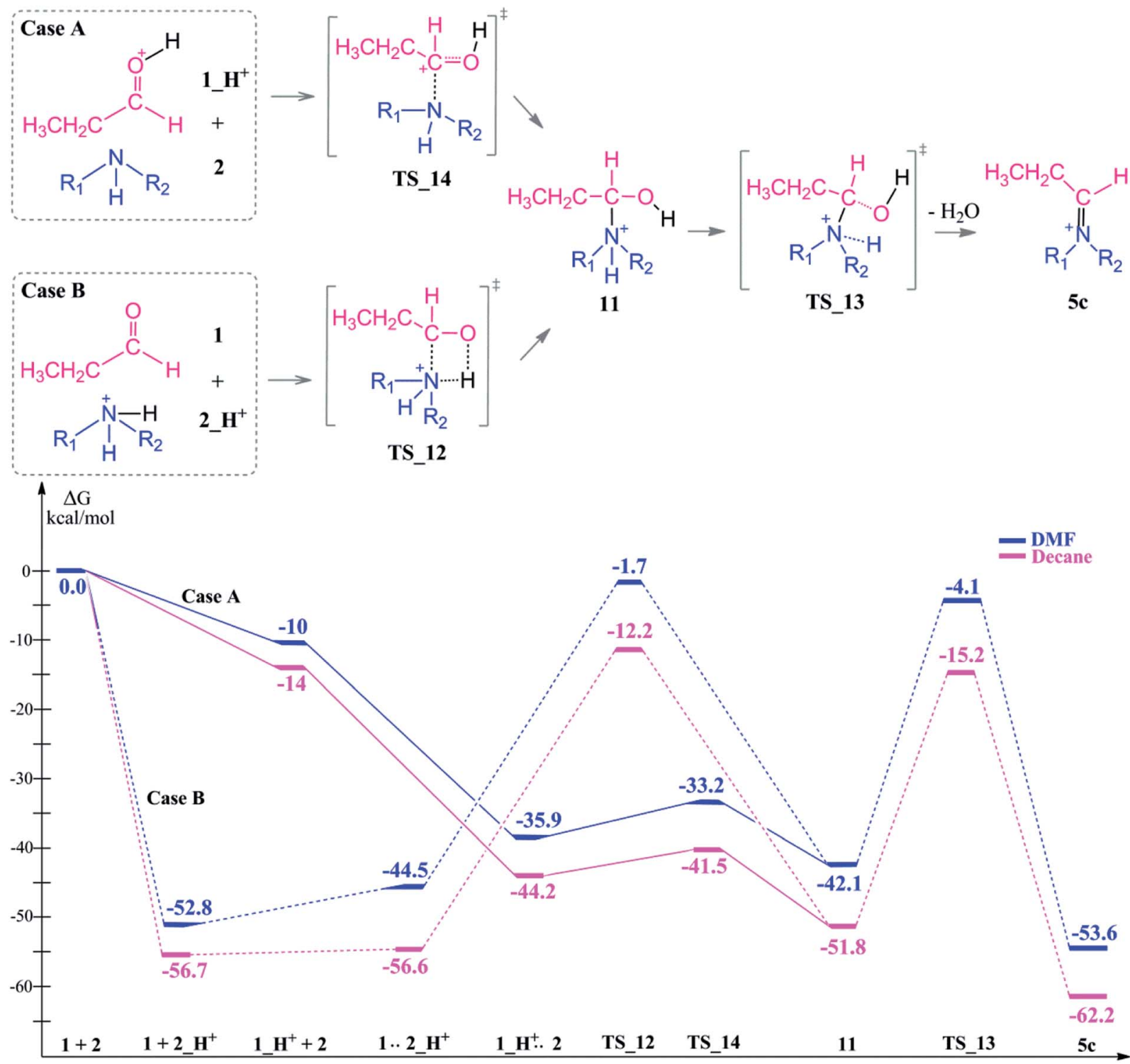

Scheme 7 Reaction mechanism including transition states and reaction intermediate structures and the free energy profile for reductive amination reaction in the presence of an acid as a co-catalyst for Case $A$ (full lines) and Case $B$ (dashed lines), $\left(R_{1}=R_{2}=-C_{2} C_{3}\right)$.

Scheme 3) is most likely to occur via this pathway and gives the final product $\mathbf{8}$.

Due to the basic character of the amine, initial protonation of the nitrogen in the presence of an acid occurs as mentioned (Case B). The calculated proton affinity to yield $2 \mathbf{H}^{+}$is around 53-57 kcal mol ${ }^{-1}$. After an intramolecular proton transfer to the carbonyl oxygen atom, the nucleophilicity of the amine is recovered. Then, the binding of the amine to the protonated aldehyde was investigated (see Scheme 7). This nucleophilic attack via TS_12 is thermodynamically possible although it has a higher transition state barrier than that of TS_14. The driving force here is the proton affinity of the amine (around $55 \mathrm{kcal} \mathrm{mol}^{-1}$ ). After the formation of intermediate 11, water elimination occurs in the same manner as discussed above.

Starting from the initially protonated reaction paths which are kinetically different, the nucleophilic additions are feasible. However, both paths are giving the same intermediate (11) in the end. Later, the reaction will go through $\mathbf{5 c}$ and yield a long chain amine as the final product.

Comparison of the neutral and acidic pathways indicates comparable transition state barrier heights (and thus similar microkinetics), although they do display strongly different thermodynamic profiles. In the presence of an acid as a cocatalyst, the reductive amination can proceed easily due to the enhanced thermodynamic stability of the iminium ion intermediate 5c. This extra stability is the thermodynamic driving force of the first reaction half before the final reduction step.

\section{Conclusion}

The mechanism of the reductive amination reaction between an aldehyde and a secondary amine to give a tertiary amine product was investigated in detail. This family of product is of great relevance to the pharmaceutical and chemical 
industries. ${ }^{1,3}$ The overall thermodynamics of the reductive amination reaction of an aldehyde was found to be exergonic by around $-15 \mathrm{kcal} \mathrm{mol}^{-1}$, independent of the carbon chain length and consideration of an organic solvent environment has a negligible effect on the free energy of the reaction.

The influence and control of the reaction by solvent properties (neutral, aqueous media, and in the presence of an acid as a co-catalyst) were investigated in detail including many different branching points.

In neutral media, the dissociation of a hydroxide ion after nucleophilic addition of the amine is clearly the preferred path and leads to an iminium as an intermediate. This is then easily hydrogenated to give the final product. In the presence of excess water, which is also released during the condensation step, the first part of the reaction is strongly affected. The formation of hydrogen bonds by explicit water coordination is increasing the electrophilicity of the aldehyde. Thus, the activation barrier for the nucleophilic addition is significantly lower and the formerly concerted amine addition becomes stepwise leading to lower energy barriers. Explicit water assistance drives the reaction pathway from an enamine- to an iminium ion-based intermediate and controls the total kinetics of the reaction process. Such insight is of relevance for the design of a suitable solvent in a large-scale production of tertiary amines from long-chain substrates from sustainable sources and the continuous removal of water during the process in order to minimize product loss due to hydrolysis.

Addition of an acid as a co-catalyst affects the entire reaction cycle, both the addition and hydrogenation steps. Keto-enol tautomerization via direct protonation of the aldehyde (Case A) can be ruled out since the formed enol has different electronic properties and loses its electrophilicity. The presence of an acid here increases the activation barrier and decelerates the rate of the reaction.

However, when first the amine is protonated (Case B), the entire pathway is significantly affected by an extra stabilization resulting from the amine's proton affinity. This protonation does not alter the barrier heights and thus the kinetics of the reaction are unchanged. However, the thermodynamic profile becomes more exothermic compared to the neutral pathway and clearly renders this pathway to become the preferred one. Thus, the presence of an acid as a co-catalyst strongly enhances the relative thermodynamic stability of the intermediate $5 \mathbf{c}$ and drives the reaction forward.

In conclusion, explicit coordination of solvent water and the co-catalyst acid assistance provide extra stabilization to different intermediates of the reductive amination of aldehydes with diethylamine and allow to guide the reaction along different pathways. Thus, by choice of solvent and $\mathrm{pH}$, the microkinetics of different steps will strongly be affected and allow the control of the kinetics and thermodynamics of the 'rate determining state'. So far, experiment did not fully resolve the structural, kinetic and thermodynamic effects of the addition of acids, bases, or water on the activity and selectivity of the catalysts. ${ }^{13}$ This computational work, for the first time, provides a detailed insight into these process control parameters and will aid the design of an appropriate reaction medium as well as a suitable catalyst for the final hydrogenation step. The choice of an appropriate transition metal-based catalyst to replace an organic hydride donor is the focus of currently ongoing work.

\section{Conflicts of interest}

There are no conflicts to declare.

\section{Acknowledgements}

We thank the Max Planck Society for the Advancement of Science for financial support and the President of the MPG for a High-Impact Scholarship to EB. This work is part of the Collaborative Research Centre "Integrated Chemical Processes in Liquid Multiphase Systems" (project A4). Financial support by the Deutsche Forschungsgemeinschaft (DFG) is gratefully acknowledged (TR 63). We gratefully acknowledge the computational source provided by the National High Performance Computing Center at Istanbul Technical University (Grant No. 5004722017) and ITU BAP (Project No. 38209).

\section{References}

1 K. S. Hayes, Appl. Catal., A, 2001, 221, 187-195.

2 A. Behr, M. Fiene, C. Buß and P. Eilbracht, Eur. J. Lipid Sci. Technol., 2000, 102, 467-471.

3 D. J. C. Constable, P. J. Dunn, J. D. Hayler, G. R. Humphrey, J. L. Leazer, R. J. Linderman, K. Lorenz, J. Manley, B. A. Pearlman, A. Wells, A. Zaks and T. Y. Zhang, Green Chem., 2007, 9, 411-420.

4 A. Behr, A. J. Vorholt, K. A. Ostrowski and T. Seidensticker, Green Chem., 2014, 16, 982-1006.

5 T. Rische and P. Eilbracht, Synthesis, 1997, 1997, 1331-1337.

6 T. G. T. A. Faßbach, M. Terhorst, A. Behr and A. J. Vorholt, ChemCatChem, 2017, 9, 1359-1362.

7 D. L. Sarah Fuchs, M. Dittmar, G. Meier, S. Heinz, A. Behr and A. J. Vorholt, ChemCatChem, 2017, 9, 1436-1441.

8 A. F. Abdel-Magid, K. G. Carson, B. D. Harris, C. A. Maryanoff and R. D. Shah, J. Org. Chem., 1996, 61, 3849-3862.

9 A. F. Abdelmagid, C. A. Maryanoff and K. G. Carson, Tetrahedron Lett., 1990, 31, 5595-5598.

10 A. F. Abdel-Magid and S. J. Mehrman, Org. Process Res. Dev., 2006, 10, 971-1031.

11 V. S. S. E. Varjosaari, P. Suating, J. J. M. Hurley, A. M. De Lio, T. M. Gilbert and M. J. Adler, Adv. Synth. Catal., 2017, 359, 1872-1878.

12 R. P. Tripathi, S. S. Verma, J. Pandey and V. K. Tiwari, Curr. Org. Chem., 2008, 12, 1093-1115.

13 S. Gomez, J. A. Peters and T. Maschmeyer, Adv. Synth. Catal., 2002, 344, 1037-1057.

14 Y. W. Qian Lei, D. Talwar, C. Wang, X. Dong and J. Xiao, Chem.-Eur. J., 2013, 19, 4021-4029.

15 T. Mizuta, S. Sakaguchi and Y. Ishii, J. Org. Chem., 2005, 70, 2195-2199.

16 C. Wang, A. Pettman, J. Bacsa and J. Xiao, Angew. Chem., Int. $E d .$, 2010, 49, 7548-7552. 
17 V. I. Tararov, R. Kadyrov, T. H. Riermeier, C. Fischer and A. Börner, Adv. Synth. Catal., 2004, 346, 561-565.

18 T. S. Sabine Pisiewicz, A.-E. Surkus, K. Junge and M. Beller, ChemCatChem, 2015, 7, 62-64.

19 V. B. O. S. Nayal, S. Sharma and N. Kumar, J. Org. Chem., 2015, 80, 5912-5918.

20 D. Seidel, Acc. Chem. Res., 2015, 48, 317-328.

21 M. T. R. Arne Dieckmann, A. Yu. Platonova, C. Zhang, D. Seidel and K. N. Houk, J. Org. Chem., 2013, 78, 4132-4144.

22 M. B. Matthew, T. Richers, A. Yu. Platonova, A. Ullrich, A. Dieckmann, K. N. Houk and D. Seidel, J. Am. Chem. Soc., 2014, 136, 6123-6135.

23 A. Trowbridge, D. Reich and M. J. Gaunt, Nature, 2018, 561, 522.

24 P. H.-Y. Cheong, C. Y. Legault, J. M. Um, N. Çelebi-Ölçüm and K. N. Houk, Chem. Rev., 2011, 111, 5042-5137.

25 N. V. Belkova, E. V. Bakhmutova-Albert, E. L. Gutsul, V. I. Bakhmutov, I. E. Golub, O. A. Filippov, L. M. Epstein, M. Peruzzini, A. Rossin, F. Zanobini and E. S. Shubinat, Inorg. Chem., 2014, 53, 1080-1090.

26 J. R. Bernardo, S. C. A. Sousa, P. R. Florindo, M. Wolff, B. Machura and A. C. Fernandes, Tetrahedron, 2013, 69, 9145-9154.

27 J. A. Fuentes, P. Wawrzyniak, G. J. Roff, M. Buhl and M. L. Clarke, Catal. Sci. Technol., 2011, 1, 431-436.

28 J. H. Gui, C. M. Pan, Y. Jin, T. Qin, J. C. Lo, B. J. Lee, S. H. Spergel, M. E. Mertzman, W. J. Pitts, T. E. La Cruz, M. A. Schmidt, N. Darvatkar, S. R. Natarajan and P. S. Baran, Science, 2015, 348, 886-891.

29 S. Moulin, H. Dentel, A. Pagnoux-Ozherelyeva, S. Gaillard, A. Poater, L. Cavallo, J. F. Lohier and J. L. Renaud, Chem.Eur. J., 2013, 19, 17881-17890.

30 J. P. Reid, L. Simon and J. M. Goodman, Acc. Chem. Res., 2016, 49, 1029-1041.

31 Y. Shibata and M. Yamanaka, J. Org. Chem., 2013, 78, 37313736.

32 L. Simon and J. M. Goodman, J. Am. Chem. Soc., 2008, 130, 8741-8747.

33 M. P. Patil and R. B. Sunoj, J. Org. Chem., 2007, 72, 82028215.

34 J. Markert, Y. Brunsch, T. Munkelt, G. Kiedorf, A. Behr, C. Hamel and A. Seidel-Morgenstern, Appl. Catal., A, 2013, 462-463, 287-295.

35 E. Schäfer, Y. Brunsch, G. Sadowski and A. Behr, Ind. Eng. Chem. Res., 2012, 51, 10296-10306.

36 A. Jörke, E. Kohls, S. Triemer, A. Seidel-Morgenstern, C. Hamel and M. Stein, Chem. Eng. Process., 2016, 102, 229-237.

37 A. Jörke, T. Gaide, A. Behr, A. Vorholt, A. Seidel-Morgenstern and C. Hamel, Chem. Eng. J., 2017, 313, 382-397.
38 A. Behr and R. Roll, J. Mol. Catal. A: Chem., 2005, 239, 180184.

39 C. Møller and M. S. Plesset, Phys. Rev., 1934, 46, 618.

40 M. J. Frisch, H. B. Schlegel, G. E. Scuseria, M. A. Robb, G. Scalmani and D. J. Fox, Gaussian 09, Gaussian, Inc., Wallingford CT, 2009.

41 K. Fukui, J. Phys. Chem., 1970, 74, 4161-4163.

42 K. Fukui, Acc. Chem. Res., 1981, 14, 363-368.

43 A. E. Reed and F. Weinhold, J. Chem. Phys., 1983, 78, 40664073.

44 A. E. Reed, R. B. Weinstock and F. Weinhold, J. Chem. Phys., 1985, 83, 735-746.

45 A. V. Marenich, C. J. Cramer and D. G. Truhlar, J. Phys. Chem. $B$, 2009, 113, 6378-6396.

46 G. Kiedorf, D. M. Hoang, A. Müller, A. Jörke, J. Markert, H. Arellano-Garcia, A. Seidel-Morgenstern and C. Hamel, Chem. Eng. Sci., 2014, 115, 31-48.

47 B. Hentschel, G. Kiedorf, M. Gerlach, C. Hamel, A. SeidelMorgenstern, H. Freund and K. Sundmacher, Ind. Eng. Chem. Res., 2015, 54, 1755-1765.

48 E. Schäfer, Y. Brunsch, G. Sadowski and A. Behr, Ind. Eng. Chem. Res., 2012, 51, 10296-10306.

49 M. Lemberg, G. Sadowski, M. Gerlach, E. Kohls, M. Stein, C. Hamel and A. Seidel-Morgenstern, AIChE J., 2017, 63, 4576-4585.

50 A.-C. Bédard, A. Adamo, K. C. Aroh, M. G. Russell, A. A. Bedermann, J. Torosian, B. Yue, K. F. Jensen and T. F. Jamison, Science, 2018, 361, 1220-1225.

51 D. S. Yufit and J. A. Howard, J. Mol. Struct., 2010, 984, 182185.

52 P. Kalck and M. Urrutigoïty, Chem. Rev., 2018, 118, 38333861.

53 Y. Hoshimoto, T. Kinoshita, S. Hazra, M. Ohashi and S. Ogoshi, J. Am. Chem. Soc., 2018, 140, 7292-7300.

54 Y. Wei, D. Xue, Q. Lei, C. Wang and J. Xiao, Green Chem., 2013, 15, 629-634.

55 D. Menche, J. Hassfeld, J. Li, G. Menche, A. Ritter and S. Rudolph, Org. Lett., 2006, 8, 741-744.

56 C. Solís-Calero, J. Ortega-Castro, A. Hernández-Laguna and F. Munoz, Theor. Chem. Acc., 2012, 131, 1263.

57 B. Szefczyk, P. Kedzierski and W. A. Sokalski, Mol. Phys., 2006, 104, 2203-2211.

58 E. Erdtman, E. A. Bushnell, J. W. Gauld and L. A. Eriksson, Comput. Theor. Chem., 2011, 963, 479-489.

$59 \mathrm{~V}$. B. Kharitonov, M. Makarova, M. A. Arsenov, Y. V. Nelyubina, O. Chusova, A. S. Peregudov, S. S. Zlotskii, D. Chusov and D. A. Loginov, Organometallics, 2018, 37, 2553-2562. 